\title{
A TRADUÇÃO/LOCALIZAÇÃO DE VIDEOGAMES: UM MAPEAMENTO DAS PESQUISAS REALIZADAS EM INSTITUIÇÕES DE ENSINO SUPERIOR BRASILEIRAS ENTRE 1998 E 2018
}

\author{
TRANSLATION/LOCALIZATION OF VIDEOGAMES: \\ MAPPING THE RESEARCH CARRIED OUT IN BRAZILIAN UNIVERSITIES \\ BETWEEN 1998 AND 2018
}

\author{
Luiz Gustavo Nogueira BARCELOS \\ Universidade de Brasília \\ Programa de Pós-Graduação em Estudos da Tradução \\ Brasília, Distrito Federal, Brasil. \\ orcid.org/0000-0002-2868-9626 \\ barcelos_gustavo@hotmail.com
}

\author{
Gleiton MALTA \\ Universidade de Brasília \\ Instituto de Letras \\ Departamento de Letras Estrangeiras e Tradução \\ Brasília, Distrito Federal, Brasil. \\ orcid.org/0000-0003-0359-4046 \\ gleitonmalta@gmail.com
}

Resumo: A localização é um tema que vem ganhando força em vista da globalização e da necessidade mercadológica de os produtos chegarem a um novo mercado de modo que não apresentem características internacionalistas e que possam interferir na sua recepção na cultura de chegada. O presente artigo integra os estudos propostos pelo grupo de Mapeamento em Tradução (MapTrad), que, além do estudo aqui proposto, desenvolve outras pesquisas, expandindo os estudos propostos inicialmente por Pagano e Vasconcellos (2003) e Alves e Vasconcellos (2013). Este estudo, inserido no campo disciplinar dos Estudos da Tradução, no seu ramo puro, descritivo e orientado à função, tem por objetivo apresentar um mapeamento dos trabalhos acadêmicos de Instituições de Ensino Superior (IES) brasileiras cujo enfoque esteja relacionado às pesquisas direcionadas às práticas de localização e tradução de videogames, no período compreendido entre 1998 e 2018. Para tanto, replicou-se parcialmente a metodologia proposta por Pagano e Vasconcellos (2003) e Alves e Vasconcellos (2016), cujos trabalhos apresentam mapeamentos relacionados aos Estudos da Tradução no Brasil. Justifica-se o trabalho pela necessidade de se levantar o status quo da produção acadêmica brasileira, em nível de mestrado e doutorado, acerca da temática, descobrindo nichos de pesquisa ainda não tão explorados e apontando temas relevantes para estudos acadêmicos dialogando com o mercado. Como resultado, constatou-se um déficit de estudos que tenham como objeto de pesquisa a localização de videogames e que, embora a quantidade de estudos relacionados à tradução tenha crescido, o mesmo não se pode afirmar quanto à tradução/localização de videogames.

Palavras-Chave: Mapeamento. Localização. Tradução. Videogames. Globalização. MapTrad.

\begin{abstract}
Localization has gained momentum because of globalization and market needs to provide products that are more oriented to the target culture in different countries and, therefore, more easily embraced by the target audience. This article integrates the studies proposed by the research group Mapping in Translation (MapTrad), which has carried out research aiming to expand the studies originally reported in Pagano and Vasconcellos (2003) and Alves and Vasconcellos (2013). As a purely descriptive, function-oriented study within Translation Studies, it aims to report on a mapping of the academic production on the localization and translation of video games that is linked to Brazilian higher education institutions (HEI) in the period from 1998 through 2018. It is based on the methodology proposed by Pagano and Vasconcellos (2003) and replicated by Alves and Vasconcellos (2016), all of whom mapped the Translation Studies in Brazil. This study is relevant to o uso irrestrito, a distribuição e reprodução em qualquer meio desde que o artigo original seja devidamente citado.
\end{abstract}


understand the status of the Brazilian academic production on the subject at both master's and doctorate levels, to identify research niches that have not been explored, and to promote a dialogue between the academia and the market. The results show a deficit of studies on the localization of videogames despite the increased number of studies on translation in general.

Keywords: Mapping. Localization. Translation. Video games. Globalization. MapTrad.

If I'm selling to you, I speak your language. If I'm buying, dann müssen Sie Deutsch sprechen! ${ }^{1}$

Willy Brandt

\section{Introdução}

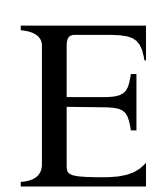

mbora tradução e localização não sejam conceitos divergentes, é importante destacar as diferenças entre os dois. De acordo com Newmark (1988, 2001), tradução pode ser definida como "[...] traduzir o significado de um texto em uma outra língua da mesma maneira que autor imprimiu em seu texto" ${ }^{2}$. Ou seja, a tradução encontra, muitas vezes, seu enfoque no nível textual, levando em consideração, a rigor, o contexto e o objetivo do texto a ser traduzido. Já a localização, como explica Schäler (1998, p. 161),

[...] pode ser definida como a adaptação linguística e cultural do conteúdo digital às condições e ao local de um mercado estrangeiro, bem como a provisão de serviços e tecnologias para a manutenção do multilinguísmo através do fluxo de informação digital global. ${ }^{3}$

Ao tratarmos de práticas de localização, consideramos, majoritariamente, o contexto cultural para o qual a informação será localizada ${ }^{4}$ - seja o conteúdo digital ou não. Dessa forma, podemos considerar que a localização é uma prática comumente voltada para um mercado específico - uma vez que seu objetivo principal é adaptar as informações contidas no material original de forma que se torne mais atraente para o público-alvo. Nesse âmbito, um dos mercados mais rentáveis é o da indústria de videogames.

A crescente busca dos(as) brasileiros(as) por esse setor de entretenimento, consequentemente, deveria refletir em pesquisas com enfoque em práticas de localização no país. Afinal, o referido mercado, ainda em fase de consolidação, apresenta diferentes possibilidades de análise dentre os mais variados jogos eletrônicos que podem ser explorados em seus diversos aspectos.

Este estudo, inserido no campo disciplinar dos Estudos da Tradução, em seu ramo teórico descritivo, faz parte de um conjunto de estudos empreendidos pelo grupo de pesquisa MapTrad - Mapeamentos em Tradução ${ }^{5}$ - do CNPq e certificado pela Universidade de 
Brasília. Além do mapeamento aqui apresentado, o grupo também está mapeando trabalhos sobre tradução médica, didática da tradução e tradução de Libras <> português, bem como ampliando os trabalhos de mapeamento já realizados por Pagano e Vasconcellos (2003) e Alves e Vasconcellos (2013).

O objetivo deste artigo é apresentar o mapeamento da produção acadêmica das Instituições de Ensino Superior (IES) brasileiras, no período de 1998 a 2018, por meio da identificação de teses e dissertações em nível de mestrado e doutorado cujo tema trate da localização de videogames de qualquer idioma para o português brasileiro e/ou das práticas de localização de videogames no Brasil. Esse período é emblemático por utilizar como recorte o período de surgimento do primeiro jogo 100\% localizado (Baldur's Gate - Bioware/Interplay em 1998) até 2018, ano em que a coleta de informações foi realizada. Este trabalho se justifica pela escassez de estudo na área, como poderá ser visto na seção 4, e por conta do apelo comercial e econômico que o tema gera.

Este trabalho está divido em cinco seções, incluindo esta Introdução. A segunda seção está dedicada a um breve preâmbulo sobre localização e os números do mercado referentes ao entretimento em questão, ou seja, os videogames. A metodologia é tema da terceira seção, na qual se explicita como os dados foram coletados e organizados, conforme metodologia parcialmente replicada de Pagano e Vasconcellos (2003) e Alves e Vasconcellos (2013). A apresentação dos dados está disposta na quarta seção, cujo conteúdo está subdividido em duas subseções: uma dedicada aos dados quantitativos e outra aos dados qualitativos e à discussão. O artigo é finalizado na quinta seção, com as considerações finais.

\section{Localização}

Ao relacionarmos o conceito de localização a um dos ramos de entretenimento mais lucrativos do mundo ${ }^{6}$, primeiramente devemos entender como a localização é aplicada a um jogo eletrônico. Com a consolidação da indústria de videogames a partir da década de 1980 e o subsequente estabelecimento do mercado europeu a partir de 1990, o processo de localização começou a passar do nível parcial (e.g., manuais técnicos, informações textuais em geral) para um processo de localização "completo":

Durante essa década, alguns jogos superaram os limites, provando, indubitavelmente, o que poderia ser feito em localização. Baldur's Gate (Bioware/Interplay 1998), por exemplo, foi um dos primeiros RPGs a ser "totalmente" traduzido e dublado em espanhol. Isso foi muito significativo do ponto 
de vista da localização porque, na maioria dos RPGs, há centenas de milhares de palavras (parte de livros, mapas e pergaminhos encontrados ao longo da aventura) a serem traduzidas, assim como milhares de arquivos de voice-over a serem gravados para cada versão da língua. Ao final dos anos 1990, o faturamento da indústria de games dobrou; e embora tenha ocorrido um crescimento interno, mais da metade dele veio dos investimentos em localização. Essa tendência continuou na década seguinte. $^{7}$ (BERNAL-MERINO, 2011, p. 15-16)

A tendência de localizar jogos eletrônicos, de fato, se tornou uma prática comum e rentável a partir dos anos 2000. Empresas como a Blizzard Entertainment, responsável por criar títulos de sucesso como Overwatch (2016) e Diablo III (2012), e a Riot Games, desenvolvedora do famoso League of Legends (2009), são algumas das empresas que exploraram em larga escala o papel da localização como um fator determinante para conquistarem o público-alvo. O público brasileiro se mostrou um dos consumidores mais aficionados por videogames no mundo. Uma pesquisa previa que, em 2018, os(as) 75,7 milhões de jogadores(as) brasileiros(as) gastariam 1,5 bilhões (em jogos eletrônicos), colocando o Brasil em $13^{\circ}$ terceiro lugar no ranking mundial de consumidores(as) de videogames (BRAZIL..., 2018).

Figura 1 - Visão geral do consumo dos(as) jogadores(as) de videogame no Brasil até o final de 2018

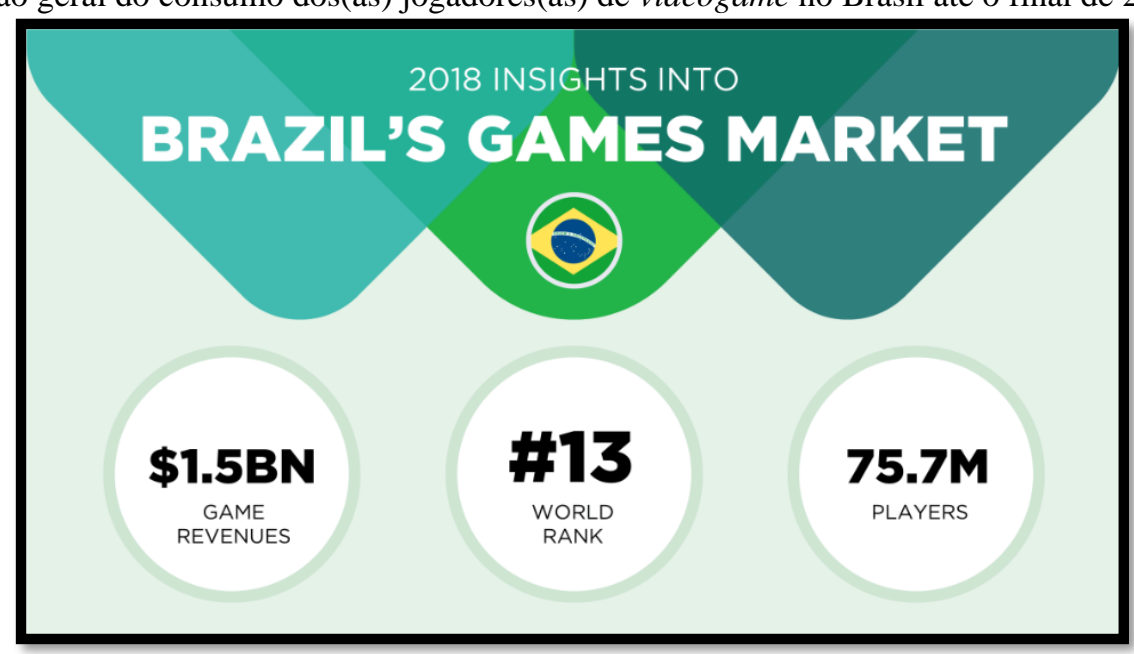

Fonte: BRAZIL..., 2018.

Diante de números tão expressivos, era de se esperar que a pesquisa na área de localização dentro do campo disciplinar dos Estudos da Tradução também seguisse o mesmo ritmo, porém, não foi o que aconteceu, como será mostrado neste estudo. Essa falta de sincronia entre mercado e academia parece ser paradoxal, já que se esperava, segundo Coletti e Mota (2013), que a necessidade de se localizarem os videogames colocassem os(as) tradutores(as)/localizadores(as) em posição de destaque. 
A escassez de estudos acadêmicos também já fora aventada por Malta e Barcelos (2018), cujo trabalho buscou analisar as características inerentes à localização de um videogame específico. Porém, na elaboração do presente estudo, foi possível comprovar que, embora haja trabalhos em nível de graduação que se desdobram em uns poucos e ainda tímidos artigos acadêmicos, a pesquisa relacionada à localização de videogames parece não achar muito espaço nos níveis de mestrado e doutorado. Tal fenômeno também merece um olhar mais atento dos(as) pesquisadores(as) da área de tradução, haja vista que o tema envolve não só questões de localização em si, mas também de globalização e mercado, o que atinge diretamente tradutores(as) já formados(as) e em formação, além dos próprios(as) pesquisadores(as).

Saldanha e Baker (2009) consideram a localização como o oposto da globalização. Enquanto esta busca intensificar as relações sociais no mundo, aquela busca dar cor local ao fluxo da informação global veiculada. Buscar estudar esse fenômeno sob o viés dos Estudos da Tradução, com foco, primeiro, na necessária descrição de produtos tradutórios envolvendo a localização e, depois, na reflexão advinda da referida descrição, é importante para que novas teorias no ramo teórico parcial do campo disciplinar ganhem fôlego, inclusive para que sejam aventadas hipóteses sobre os procedimentos em vigor tanto em nível internacional como em níveis regional, nacional e local.

\section{Metodologia}

Para realizar o mapeamento aqui proposto, como anunciado anteriormente, este estudo replica parcialmente a metodologia utilizada por Pagano e Vasconcellos (2003), que, por sua vez, inspirou o trabalho de Alves e Vasconcellos (2016). No primeiro caso, as autoras realizaram um mapeamento dos Estudos da Tradução no Brasil no período de 1998 a 2008. Baseadas no Mapa Conceitual de Holmes (1972/1988), as autoras propõem um mapa brasileiro, cujos ramos foram expandidos quando relacionados ao primeiro mapa, ou seja, o de Holmes.

Alves e Vasconcellos (2013), por sua vez, realizaram um levantamento das teses e dissertações brasileiras, cujo tema é a tradução, durante o período de 2006 a 2010. Os autores destacaram trabalhos cujos temas envolviam o uso da Linguística de Corpus e a interface com a Linguística Sistêmico-Funcional.

O presente estudo levou em consideração todas as IES mapeadas pelos dois estudos anteriores (30 no total), porém, direcionando as pesquisas para a tradução/localização de 
videogames. Ademais, ampliou o período de tempo, de 1998 a 2018. O Quadro 1 mostra as instituições consideradas nos estudos de Pagano e Vasconcellos (2003) e de Alves e Vasconcellos (2013), respectivamente.

Quadro 1 - Instituições de Ensino Superior mapeadas em Pagano e Vasconcellos (2003) e em Alves e Vasconcellos (2013)

\begin{tabular}{|c|c|}
\hline Estudo & IES \\
\hline Pagano e Vasconcellos (2003) & $\begin{array}{l}\text { Universidade Federal de Minas Gerais (UFMG) } \\
\text { Universidade Federal de Santa Catarina (UFSC) } \\
\text { Universidade Federal do Rio de Janeiro (UFRJ) } \\
\text { Universidade Federal do Rio Grande do Sul (UFRGS) } \\
\text { Universidade de São Paulo (USP) } \\
\text { Universidade Estadual de Campinas (UNICAMP) } \\
\text { Universidade Estadual Paulista (UNESP) } \\
\text { Universidade Estadual do Ceará (UECE) } \\
\text { Pontifica Universidade Católica de São Paulo (PUC-SP) } \\
\text { Pontifica Universidade Católica do Rio de Janeiro (PUC-Rio) } \\
\text { Pontifica Universidade Católica do Rio Grande do Sul (PUC-RS) }\end{array}$ \\
\hline Alves e Vasconcellos (2016) & $\begin{array}{l}\text { Universidade de Brasília (UnB) } \\
\text { Universidade Federal Fluminense (UFF) } \\
\text { Universidade do Estado do Rio de Janeiro (UERJ) } \\
\text { Universidade Federal do Espírito Santo (UFES) } \\
\text { Universidade Estadual da Paraíba (UEPB) } \\
\text { Universidade Federal de Juiz de Fora (UFJF) } \\
\text { Universidade Federal de Santa Maria (UFSM) } \\
\text { Pontifica Universidade Católica de Minas Gerais (PUC-MG) } \\
\text { Universidade Federal do Ceará (UFC) } \\
\text { Mackenzie } \\
\text { Universidade de Caxias do Sul (UCS) } \\
\text { Universidade Estadual de Maringá (UEM) } \\
\text { Universidade Federal de Alagoas (UFAL) } \\
\text { Universidade Federal da Bahia (UFBA) } \\
\text { Universidade Federal de Mato Grosso do Sul (UFMS) } \\
\text { Universidade Federal do Rio Grande do Norte (UFRN) } \\
\text { Universidade Gama Filho (UGF) } \\
\text { Universidade Estadual do Oeste do Paraná (UNIOESTE) } \\
\text { Universidade de Sorocaba (UNISO) }\end{array}$ \\
\hline
\end{tabular}

Fonte: elaboração própria

A fim de identificar todos os trabalhos que apresentam enfoque em práticas de tradução/localização em videogames em todas as IES mencionadas, foram realizadas pesquisas individuais nos respectivos repositórios $^{8}$ acadêmicos de cada uma dessas instituições. O padrão de busca em todos os repositórios considerou apenas teses e dissertações em nível de mestrado e doutorado. Além disso, foram consideradas as seguintes palavras-chave ${ }^{9}$ para as buscas nos repositórios: video-games, videogame, videogames, video game, video games, jogo eletrônico, jogos eletrônicos, localização, tradução.

$\mathrm{Na}$ coleta das informações e dos dados, em seus aspectos tanto quantitativos quanto qualitativos, Alves e Vasconcellos (2013) utilizaram o site "Domínio Público" como fonte de 
dados para realizar suas pesquisas. Por meio desse site, puderam filtrar as pesquisas por "autor", "título", "ano da tese" etc. No estudo apresentado neste artigo, não foi possível utilizar a mesma forma de coleta, uma vez que o site não apresentou resultados produtivos para localização de videogames; portanto, optou-se por uma nova abordagem.

Para extrair o maior número de dados possível, utilizaram-se, então, os repositórios acadêmicos de cada IES que conforma esta pesquisa. Ao total, foram consideradas 30 IES que apresentam teses e dissertações em Tradução e áreas correlatas. Essas IES são aquelas já mapeadas em Pagano e Vasconcellos (2003) e em Alves e Vasconcellos (2016). A data inicial proposta é emblemática, 1998, já que naquele ano foi lançado, na Espanha, o primeiro videogame $100 \%$ localizado $^{10}$. Além disso, devido à escassez de produções acadêmicas sobre "localização de videogames" no âmbito dos Estudos da Tradução, optou-se por ampliar o recorte até o momento da pesquisa, ou seja, novembro de 2018.

O período escolhido para o cotejamento de dados é outro ponto que diferencia este estudo daqueles realizados por Pagano e Vasconcellos (2003) e por Alves e Vasconcellos (2016) - os quais apresentam um recorte temporal de cinco anos. O período aqui proposto foi ampliado para 20 anos.

\section{Apresentação dos dados}

Com base no método de Alves e Vasconcellos (2016), primeiramente serão apresentados os dados quantitativos e, em seguida, os dados qualitativos. As palavras-chave foram pesquisadas com e sem aspas a fim de ampliar os resultados obtidos, tendo em vista que a pesquisas entre aspas fornecem resultados mais precisos sobre a expressão buscada. Utilizou-se o comando "Command $+F$ " do teclado (no sistema operacional MacOS) a fim de filtrar todo o conteúdo obtido na busca por meio da palavra digitada na janela que aparece após a execução desse comando. Tal processo foi adotado em todos os repositórios acadêmicos das IES que os possuíam. No caso das instituições que desproviam de banco de dados próprio, recorria-se ao catálogo de teses e dissertações da CAPES.

Inicialmente, aventou-se a possibilidade de se utilizar o banco da BDTD (Biblioteca Digital Brasileira de Teses e Dissertações). Contudo, após as buscas iniciadas no site em questão, notou-se que não apresentava resultados sobre localização de videogames. Dessa forma, a pesquisa no referido site também foi abandonada.

Algumas das principais barreiras no processo metodológico adotado nesta pesquisa foram as divergências e heterogeneidades dos portais que serviram como banco de dados para 
a compilação do material analisado. Alguns apresentavam sistemas mais leves e fluidos, com um design desenvolvido a fim de facilitar o acesso do usuário; outros possuíam um design menos atual e apresentavam resultados divergentes em relação aos termos pesquisados, caracterizando buscas avançadas incoerentes e resultando no descarte dos dados sem relação com o tema. Como exemplo, temos o caso do repositório acadêmico da USP, que apresentava resultados divergentes ao pesquisado utilizando as palavras-chave.

Alguns sites apresentam opções de busca por palavra-chave (como o da USP), outros disponibilizam filtros por departamentos (como o da UFMG), alguns organizam a busca por período de tempo (como o repositório da UFRJ) etc. Isso gerou incongruência nos resultados das buscas, que foram realizadas de maneira específica em cada repositório. Tais incongruências se davam em alguns resultados que apresentavam material menos específico, isto é, que divergem do campo do saber em tela, levando a um número maior de teses e dissertações. Em contrapartida, outros sites, cujo filtro era aplicado de forma mais eficaz, apresentavam resultados mais precisos. Não obstante, foi possível subtrair os dados inconsistentes para então realizar a análise.

134 Dentre os resultados, a busca pela palavra-chave "tradução", em sua maioria, apresentou mais resultados, como já era esperado. Ademais, em repositórios nos quais não foi possível filtrar por "áreas do conhecimento" (e.g., UFMG), o termo "tradução" aparecia em outras áreas, como Biologia e Ciências da Saúde. O termo "localização" apenas se associava à grande área de Tradução quando envolvia a temática de videogames; nos resultados restantes, a pesquisa era direcionada para qualquer outra área que não fosse relevante para este mapeamento (e.g., Geografia). Os termos restantes - "videogames" e suas variantes, "games" e suas variantes; e "jogos" e suas variantes - apresentavam estudos da Computação e áreas correlatas.

\subsection{Análise quantitativa dos dados}

Adotando a catalogação proposta no estudo de Alves e Vasconcellos (2016), as 30 IES foram organizadas em um quadro para ilustrar os dados. O Quadro 2 apresenta as instituições pesquisadas, as unidades da federação a qual pertencem, a região do Brasil para a determinação de produção por meio de estatísticas de núcleos de concentração por zona, o número de produções acadêmicas (entre teses e dissertações) e, por fim, a fonte de coleta. 
Quadro 2 - Resultado das dissertações e teses obtidas (divididas pelas IES propostas)

\begin{tabular}{|c|c|c|c|c|}
\hline Instituição & $\begin{array}{l}\text { Unidade da } \\
\text { Federação }\end{array}$ & $\begin{array}{l}\text { Região do } \\
\text { Brasil }\end{array}$ & Número & Fonte \\
\hline USP & São Paulo & Sudeste & 435 & $\begin{array}{c}\text { https://www.teses.usp.br/index.php?option=co } \\
\text { m_jumi\&fileid=19\&Itemid=87\&lang=pt- } \\
\text { br\&g=1\&b3=Tradução\&c3=p\&o3=AND }\end{array}$ \\
\hline UFSC & Santa Catarina & Sul & 383 & $\begin{array}{c}\text { http://www.pget.ufsc.br/curso/teses_e_dissertac } \\
\text { oes.php }\end{array}$ \\
\hline UFJF & Minas Gerais & Sudeste & 202 & https://repositorio.ufjf.br/jspui/ \\
\hline PUC-Rio & Rio de Janeiro & Sudeste & 157 & http://www.ctc.puc-rio.br/teses-e-dissertacoes/ \\
\hline UFMG & Minas Gerais & Sudeste & 131 & http://www.bibliotecadigital.ufmg.br/dspace/ \\
\hline UNESP & São Paulo & Sudeste & 126 & https://repositorio.unesp.br \\
\hline UFAL & Alagoas & Nordeste & 105 & http://www.repositorio.ufal.br \\
\hline UnB & Distrito Federal & Centro-Oeste & 89 & http://bdm.unb.br \\
\hline UEM & Paraná & Sul & 83 & http://nou-rau.uem.br/nou-rau/ \\
\hline UFRGS & Rio Grande do Sul & Sul & 75 & https://lume.ufrgs.br \\
\hline UFC & Ceará & Nordeste & 65 & http://www.repositorio.ufc.br/handle/riufc/3616 \\
\hline UFRJ & Rio de Janeiro & Sudeste & 63 & https://pantheon.ufrj.br \\
\hline UFRN & Rio Grande do Norte & Nordeste & 50 & https://repositorio.ufrn.br/jspui/ \\
\hline UNICAMP & São Paulo & Sudeste & 34 & http://repositorio.unicamp.br \\
\hline UFSM & Rio Grande do Sul & Sul & 30 & https://repositorio.ufsm.br \\
\hline UERJ & Rio de Janeiro & Sudeste & 21 & http://www.bdtd.uerj.br \\
\hline UNIOESTE & Paraná & Sul & 15 & http://bdtd.ibict.br/vufind/ \\
\hline UECE & Ceará & Nordeste & 13 & $\begin{array}{c}\text { http://www.uece.br/pibidhistoriafafidam/index. } \\
\text { php/repositorio-digital }\end{array}$ \\
\hline PUC-MG & Minas Gerais & Sudeste & 8 & $\begin{array}{l}\text { http://www.sistemas.pucminas.br/BDP/SilverSt } \\
\text { ream/Pages/pg_BDPPrincipal.html }\end{array}$ \\
\hline PUC-RS & Rio Grande do Sul & Sul & 8 & http://repositorio.pucrs.br/dspace/ \\
\hline UFMS & Mato Grosso do Sul & Centro-Oeste & 6 & http://repositorio.ufms.br:8080/jspui/ \\
\hline UFES & Espírito Santo & Sudeste & 5 & http://repositorio.ufes.br \\
\hline UCS & Rio Grande do Sul & Sudeste & 4 & https://repositorio.ucs.br/xmlui/ \\
\hline PUC-SP & São Paulo & Sudeste & 3 & https://tede2.pucsp.br \\
\hline UFF & Rio de Janeiro & Sudeste & 1 & https://app.uff.br/riuff/ \\
\hline UEPB & Paraíba & Nordeste & 0 & http://dspace.bc.uepb.edu.br/jspui/ \\
\hline $\begin{array}{c}\text { UPM } \\
\text { (Mackenzie) }\end{array}$ & São Paulo & Sudeste & 0 & $\begin{array}{l}\text { http://pergamum.mackenzie.br/biblioteca/index. } \\
\text { php }\end{array}$ \\
\hline UFBA & Bahia & Nordeste & 0 & https://repositorio.ufba.br/ri/ \\
\hline UGF & Rio de Janeiro & Sudeste & 0 & http://biblioteca.phorteeducacional.com.br \\
\hline UNISO & São Paulo & Sudeste & 0 & http://www.capes.gov.br \\
\hline
\end{tabular}

Fonte: elaboração própria.

Após o primeiro contato com as pesquisas proeminentes deste estudo, obteve-se o primeiro dado deste artigo, que são as pesquisas identificadas na busca realizada nos repositórios. Por meio desse recorte, obteve-se o resultado de 2.112 trabalhos acadêmicos, entre dissertações de mestrado, teses de doutorado e livre docência sobre temas de tradução (não necessariamente de localização), publicados anteriormente ao dia $1^{\circ}$ de novembro de 2018 .

É importante ressaltar que este estudo considera apenas as teses e dissertações produzidas sob a temática "localização de videogames", que está inserida dentro dos Estudos da Tradução. O Quadro 2 ilustra a dificuldade do mapeamento deste estudo - caracterizada 
pela diferença logística de armazenamento e quantidade de pesquisas em cada repositório acadêmico. Alguns permitiram um refinamento mais adequado; outros, ao passo que apresentavam mais dados, distanciavam-se do conceito de "localização de videogames". Utilizou-se, também, o catálogo de teses e dissertações da CAPES para a pesquisa, tendo em conta que a IES UNISO não possuía site próprio de repositório acadêmico.

Após o levantamento dos dados, foram organizadas as teses e dissertações que apresentavam, exclusivamente, o tema "localização de videogames" como objeto de pesquisa. Dessa forma, dentre os trabalhos acadêmicos coletados, foram selecionados apenas os que apresentavam enfoque em localização. Entretanto, como o enfoque desta pesquisa é localização de videogames, a dissertação de Luiz Alfredo Harris Maranesi, intitulada Estudo de um caso de localização de um software ERP de código livre, de 2011, e a dissertação de Fausto Magalhães Silveira, Terminologia e tradução na localização de software, de 2013, foram desconsideradas por não se encaixarem no propósito desta pesquisa.

Para chegar a essa constatação, foram separadas as teses e dissertações que continham as palavras-chave previamente mencionadas. Em seguida, foram acessados os resumos desses 136 trabalhos a fim de verificar se mantinham relação com o tema localização de videogames.

Além dos 2.112 trabalhos obtidos por meio dos repositórios das IES, algumas instituições apresentaram problemas em seu sistema de domínio público. As IES UNISO e UFG não apresentavam material em seus sites próprios. A UNISO optou por direcionar o usuário ao site da CAPES, o qual, contudo, não apresentou resultados segundo os critérios adotados nesta pesquisa. A UFG, por sua vez, apresentou um site próprio com o repositório acadêmico; no entanto, durante todas as tentativas de pesquisas (realizadas em 01, 05 e 06 de novembro de 2018), obteve-se uma mensagem de erro (“Whoops, looks like something went wrong") para qualquer tipo de busca.

Em relação a esta pesquisa, é possível que os resultados das buscas tenham desconsiderado algum estudo. Isso se dá porque, neste artigo em especial, houve mudança significativa na metodologia e na forma de compilação de dados, e a falta de homogeneização interfere diretamente no resultado proposto. Em diversos repositórios, foi possível encontrar textos utilizando o filtro por "teses \& dissertações"; em outros, apenas por "palavras-chave"; em alguns, apenas por uma palavra que poderia estar no assunto, tema, resumo ou título do trabalho em si. Os resultados são os apresentados no Gráfico 1. 
Gráfico 1 - Instituições de Ensino Superior e número de resultados obtidos com as palavras-chave utilizadas

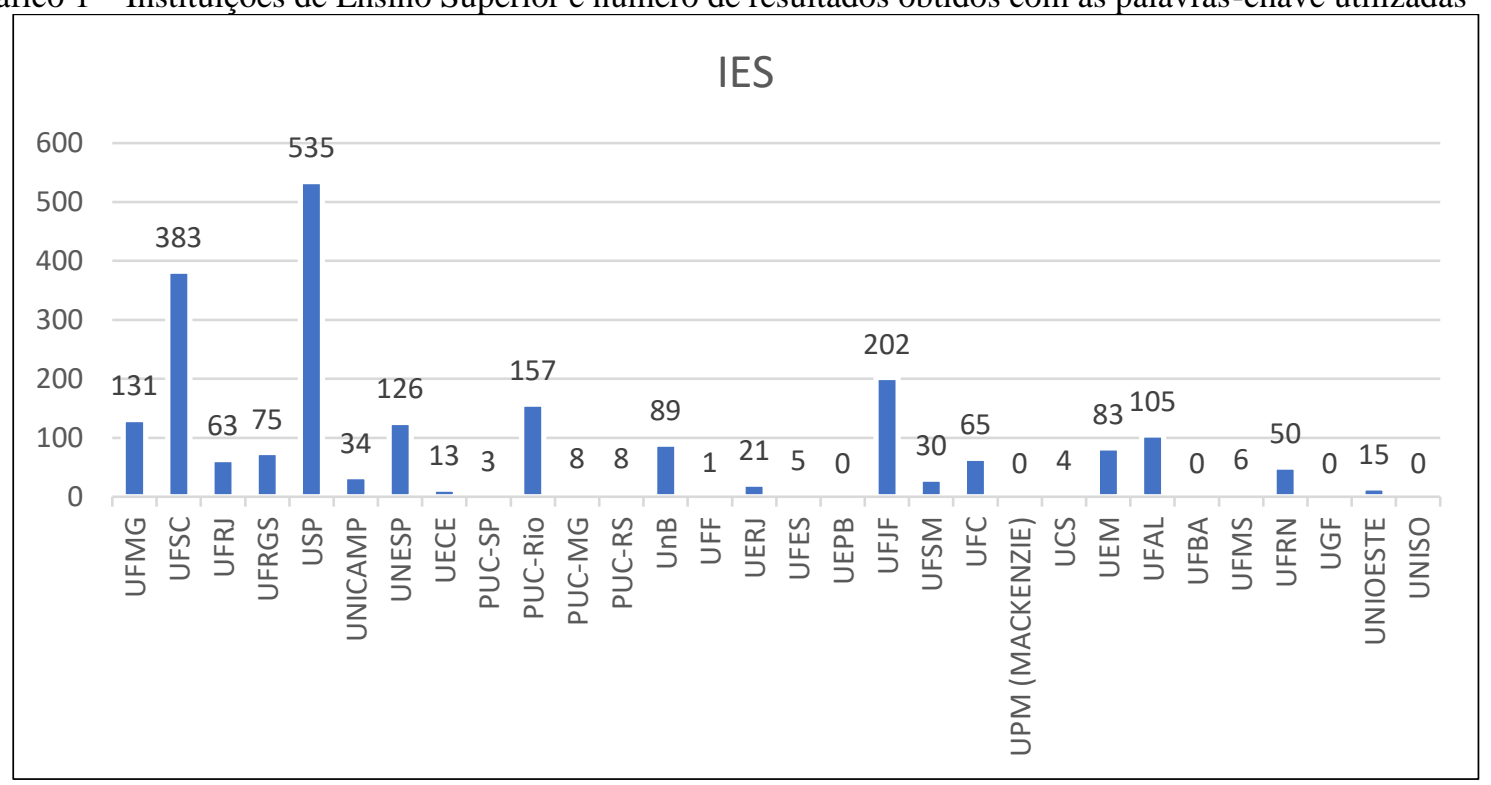

Fonte: elaboração própria.

O Gráfico 1 mostra os resultados obtidos nas buscas realizadas. A linha lateral esquerda (eixo y) determina a quantidade de resultados obtidos mediante o filtro das seguintes palavras-chave: video-game; video-games; videogame; videogames; video game; video games; jogo eletrônico; jogos eletrônicos; localização; localização de games; e tradução. A linha inferior (eixo x) se refere a cada IES, sendo possível observar, destacadamente, que a USP, UFSC, UFJF, PUC-RIO, UFMG, UNESP, UFAL e UnB são as IES que mais apresentaram resultados nas pesquisas realizadas.

O Quadro 3 apresenta os trabalhos catalogados na área de localização de videogames com dados de autoria, título, IES em que foi desenvolvido, ano de publicação e o nível mestrado e/ou doutorado.

Quadro 3 - Teses e dissertações de localização de games

\begin{tabular}{|l|c|c|c|c|}
\hline \multicolumn{1}{|c|}{ Autor } & \multicolumn{1}{c|}{ Título } & IES & $\begin{array}{c}\text { Ano de } \\
\text { publicação }\end{array}$ & Nível \\
\hline $\begin{array}{l}\text { Meggie Rosar } \\
\text { Fornazari }\end{array}$ & $\begin{array}{c}\text { Localization practices in trading card games: Magic } \\
\text { The Gathering from English into Portuguese }\end{array}$ & UFSC & 2014 & Mestrado \\
\hline $\begin{array}{l}\text { Ricardo Vinicius } \\
\text { Ferraz de Souza }\end{array}$ & $\begin{array}{c}\text { Tradução e videogames: uma perspectiva histórico- } \\
\text { descritiva sobre a localização de games no brasil }\end{array}$ & USP & 2015 & Mestrado \\
\hline Fernando da Silva & $\begin{array}{c}\text { The mapping of localized contents in the videogame } \\
\text { inFamous 2: A multimodal corpus-based analysis }\end{array}$ & UFSC & 2016 & Doutorado \\
\hline $\begin{array}{l}\text { Renan Abdalla } \\
\text { Leimontas }\end{array}$ & $\begin{array}{c}\text { "Eu vou equalizar sua cara": análise da localização } \\
\text { do video game Mortal Kombat X }\end{array}$ & UFSC & 2017 & Mestrado \\
\hline $\begin{array}{l}\text { Hemanoel M. } \\
\text { Sousa e Silva }\end{array}$ & $\begin{array}{c}\text { O estado da arte da localização/tradução de } \\
\text { videogames }\end{array}$ & UFC & 2018 & Mestrado \\
\hline
\end{tabular}

Fonte: elaboração própria. 
Os dados obtidos após o cotejamento inicial e o filtro utilizado, seja por palavraschave ou após análise individual, foram de cinco textos cujo enfoque se relaciona diretamente à localização de videogames. Os outros, 2.207, dividem-se em trabalhos sobre tradução (de forma geral) e localização, não especificamente de videogames.

Dessa forma, somente $0,22 \%$ de todos os trabalhos foi dedicado à localização de videogames. O número é ínfimo se comparado aos milhões de usuários de videogames no Brasil e ao mercado que o entretimento movimenta tanto em cifras relacionadas a capital como a nicho em mercado de trabalho para tradutores(as)/localizadores(as).

A produção de estudos sobre o tema divididos entre as IES brasileiras pode ser visualizada no Gráfico 2.

Gráfico 2 - Teses e dissertações sobre "localização de videogames"

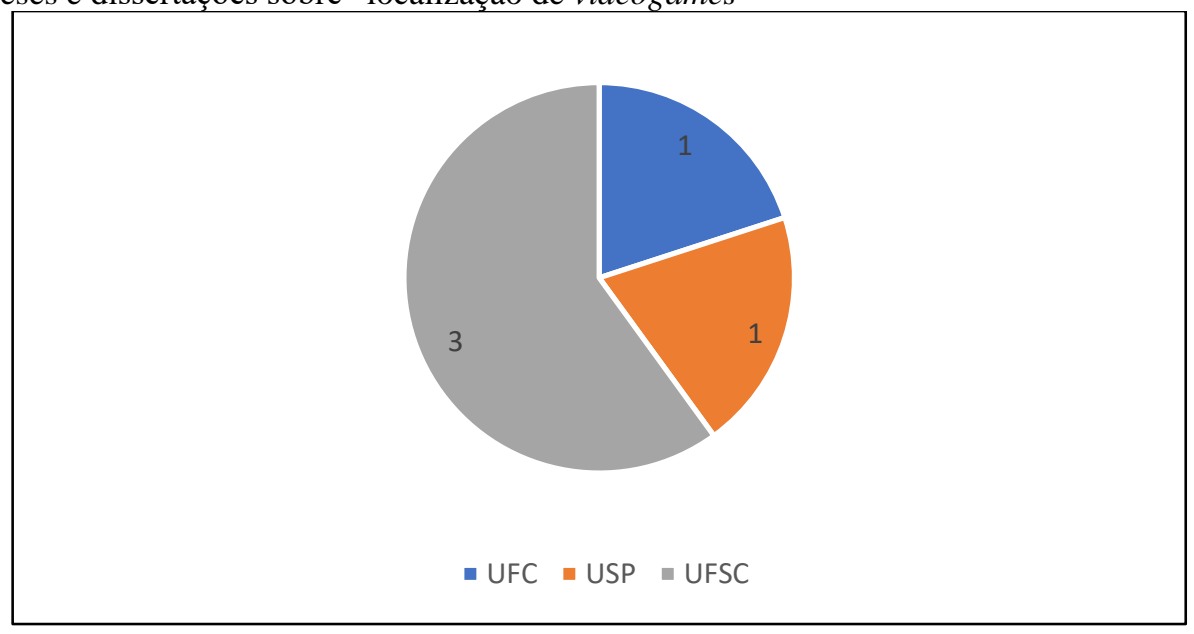

Fonte: elaboração própria.

Conforme mostrado no Gráfico 2, nota-se que, em termos de "localização de videogames", a UFSC é a maior produtora de teses e dissertações na área - apresentando três produções acadêmicas publicadas no site de seu repositório. Em seguida, a USP e a UFC aparecem com uma publicação cada.

A baixa produção acadêmica destoa da grande demanda comercial que o tema envolve. Dessa forma, nota-se um grande distanciamento entre a academia e a indústria de localização no Brasil, tema relevante para um estudo futuro, principalmente no que se refere ao porquê da falta de interesse dos estudiosos brasileiros filiados ao campo disciplinar dos Estudos da Tradução em se aprofundar no tema. 


\subsection{Análise Qualitativa dos dados}

Como mencionado anteriormente, o número de produções cujo objeto de estudo se relaciona com localização de videogames não chega a $0,3 \%$ de toda a produção acadêmica na área de Tradução. Verifica-se, nos trabalhos de Pagano e Vasconcellos (2003) e de Alves e Vasconcellos (2016), que o número de estudos produzidos nas áreas correlatas à Tradução vem crescendo. No entanto, apesar do aumento do volume de pesquisas na área, poucos estudos apresentam enfoque acadêmico direcionado para a localização de videogames. Nessa análise direta, o número de teses e dissertações que abordam essa temática é escasso, se colocado em contraste com as demais áreas dos Estudos da Tradução.

Em direção oposta aos estudos que deram origem a esta réplica - Pagano e Vasconcellos (2003); Alves e Pagano (2016) -, a proposta deste artigo foi mapear apenas um recorte dentro dos Estudos da Tradução. Considerando-se isso, não foi realizada a mesma análise proposta nos estudos mencionados, nos quais foram observadas e determinadas as produções acadêmicas (e seu crescimento) em cada IES participante desta pesquisa. Neste artigo, em particular, o enfoque foi apresentar a produção acadêmica sobre o tema tradução/localização de videogames. Nesse sentido, o passo seguinte foi analisar os trabalhos apresentados e mapear os pontos de concentração de interesse dentro do tema abordado.

Inicialmente, foi analisado a dissertação de Meggie Rosar Fornazari (2014), intitulada "Localization practices in trading card games: Magic The Gathering from English into portuguese", orientada pelo Prof. Dr. Lincohn Fernandes. A pesquisa tem como objeto de estudo o jogo de cartas/estampas colecionáveis Magic the Gathering ${ }^{11}$, que, apesar de não se tratar de um game digital, apresenta uma versão para computador intitulada Magic Arena que utiliza essas traduções. A autora utiliza corpus paralelo bilíngue para observar restrições técnicas e políticas de localização de 249 cartas, das quais os textos foram extraídos utilizando as ferramentas Wordsmith 3.0 e Notepad++. Apresentou como resultado que o produto tradutório é linguisticamente adequado, mas não é aceitável ao sistema cultural do país.

O segundo trabalho analisado foi a dissertação de Ricardo Vinicius Ferraz de Souza (2015), cujo título é Tradução e Videogames: uma perspectiva histórico-descritiva sobre a localização de games no Brasil, orientada pelo Prof. Dr. John Milton. Esse é um trabalho historiográfico que tem como objetivo a análise e descrição da relação histórica entre tradução e localização de games, traçando um panorama do contexto histórico brasileiro.

Cronologicamente, verificou-se a tese de doutorado de Fernando da Silva (2016), orientado pela Profa. Dra. Viviane Maria Heberle: The mapping of localized contents in the 
videogame inFamous 2: a multimodal corpus-based analysis. O trabalho analisa a prática da localização de videogames à luz dos Estudos da Tradução por meio da análise de um corpus multimodal, tendo como principal objeto de estudos o jogo inFamous 2 para Playstation 3, em sua versão localizada para o português brasileiro. A metodologia se baseou na observação dos aspectos terminológicos e percepção cultural entre os pares linguísticos (inglês - português). Como resultado, o autor verificou que o sistema de anotação utilizado apresentou pequenas mudanças em termos de uso da linguagem avaliativa e que o perfil lexical nas linhas de diálogos apresentou atenuação na prosódia semântica.

Renan Abdalla Leimontas (2017) submeteu a dissertação intitulada "Eu vou equalizar sua cara": Análise da localização do video game Mortal Kombat X, orientada pelo Prof. Dr. Gilles Jean Abes. O autor buscou discorrer sobre a localização do jogo Mortal Kombat X e afirma que a localização não foi tão bem recebida pelo público-alvo. Utilizou-se da óptica dos Estudos da Tradução para analisar a localização, apontando os problemas discutidos pela comunidade. Metodologicamente, o autor coletou os dados fazendo uso do modo de jogo "Luta Única", analisando as falas dos personagens sob os critérios apresentados por Chandler 140 e Deming (2012). Como resultado, o autor apresentou 40 amostras de diálogos que formam uma base para as críticas dos fãs do game.

Por fim, a dissertação $O$ estado da arte da localização/tradução de videogames, de Hemanoel Mariano Sousa e Silva (2018), orientado pelo Prof. Dr. Rafael Ferreira da Silva, analisou o panorama atual da localização de videogames, traçando-o historicamente e identificando suas características. $\mathrm{O}$ autor também evidencia a escassez de pesquisas na área, tal qual foi mostrado nos resultados do mapeamento aqui proposto. Para isso, foram analisados alguns jogos em perspectiva descritiva e histórica, além de conceitos de gameplay de Adams (2010), Novak (2012), Rollings e Morris (2010) etc.

Os trabalhos elencados trazem discussões pertinentes à área da localização de videogames. Esse mapeamento permitiu que fosse possível a enumeração da quantidade de produção acadêmica a respeito da temática investigada. Esse número comprova que a baixa incidência de pesquisas sobre localização traz imediatamente a necessidade de a academia percebê-la como tema relevante de estudo e propor uma visão sobre a temática. Ademais, não foi possível detectar nos estudos analisados questionamentos sobre o papel e o conceito de localização em si, levando-se em conta o contexto cultural brasileiro. Na maioria das vezes, o termo - localização - é recebido como pronto e não sofre qualquer problematização. 


\section{Considerações finais}

Há diversos fatores que podem acarretar a falta de interesse de pesquisadores(as) da área de tradução/localização de videogames. Um desses fatores está relacionado diretamente ao quão recente o conceito de localização de videogames está presente na academia. A localização completa do primeiro videogame foi realizada nos anos 1990 - o clássico Baldur's Gate (Bioware/Interplay). Ademais, a prática de tradução de jogos eletrônicos não era uma área muito "aquecida" no país, recebendo a reconhecida visibilidade apenas recentemente ${ }^{12}$. Tal tendência começou a mudar por conta da expansão do mercado nacional de videogames ${ }^{13}$, já que o Brasil é o maior consumidor deste tipo de mídia na América Latina e tem potencial para se tornar o $13^{\circ}$ maior do mundo.

Além disso, a forma como tais traduções e/ou localizações eram feitas também está passando por mudanças. O mercado está mais exigente, e as empresas estão investindo cada vez mais em profissionais em tradução para localizarem seus jogos para o mercado nacional ${ }^{14}$.

Toda essa efervescência movimenta e cria uma identidade para a localização de games no Brasil. Com isso, é esperado que surjam pesquisas e pesquisadores que desejem investigar e se aprofundar mais nessa área do campo tradutório. Talvez por isso, no recorte utilizado na pesquisa (1998-2018), nota-se que as poucas produções acadêmicas com esse enfoque começaram a surgir a partir de 2014. Também é possível notar produções de TCCs (Trabalhos de Conclusão de Curso) que abordam tal temática, além de artigos acadêmicos não incluídos neste estudo - o que reforça ainda mais a teoria de que o entusiasmo em relação a esse mercado pode fomentar a pesquisa no/para o setor.

O mapeamento proposto não determina ou conclui uma ideia, mas promove a reflexão e o debate sobre a necessidade de novas pesquisas sobre a tradução/localização de games no país. O fato de ser uma área nova, que segue em crescimento, corrobora a ideia de inevitabilidade de futuras investigações. Sugere-se, portanto, a continuidade deste trabalho, utilizando configurações individuais dentro dos Estudos da Tradução, para que sejam mapeadas, além do conteúdo geral, também as subáreas de pesquisas dentro do campo disciplinar, o que já está sendo desenvolvido pelo grupo de pesquisa aqui representado (MapTrad). Destarte, o mapeamento pode ser mais específico e com base nos interesses de cada pesquisador(a) em estudos posteriores. 


\section{REFERÊNCIAS}

ADAMS, Ernest. Fundamentals of game design. 2. ed. Berkeley: New Riders, 2009.

ALVES, Daniel; VASCONCELLOS, Maria Lúcia. Metodologias de pesquisa em Estudos da Tradução: uma análise bibliométrica de teses e dissertações produzidas no Brasil entre 20062010. D.E.L.T.A., São Paulo, v. 32, n. 2, p. 375-404, 2016. doi: https://doi.org/10.1590/01024450827796709063513

BAKER, Mona; SALDANHA, Gabriela. Routledge Encyclopedia of Translation Studies. New York: Routledge, 2009. doi: https://doi.org/10.4324/9780203872062

BERNAL-MERINO, Miguel Á. A brief history of game localisation. TRANS. Revista de Traductología, Málaga, n. 15, p. 11-17, 2011. doi: https://doi.org/10.24310/TRANS.2011.v0i15.3191

BRAZIL games market 2018. Newzoo, 6 jul. 2018. Disponível em: https://newzoo.com/insights/infographics/brazil-games-market-2018/. Acesso em: 2 mar. 2018.

CHANDLER, Heather Maxwell; DEMING, Stephanie O'Malley. The Game Localization Handbook. 2. ed. Sudbury: Jones \& Bartlett Learning, 2012.

142 COLETTI, Bruna Luizi; MOTTA, Lennon. A localização de games no Brasil - Um ponto de vista prático. In-traduções, Florianópolis, v. 0, n. 5, p. 1-12, nov. 2013.

FORNAZARI, Meggie Rosar. Localization practices in trading card games: Magic The Gathering from English into Portuguese. 2014. Dissertação (Mestrado em Inglês: Estudos Linguísticos e Literários) - Universidade Federal de Santa Catarina (UFSC), Florianópolis, 2014.

HOLMES, James S. The name and nature of translation studies. In: VENUTI, L. (Ed.) The Translation Studies Reader. 2. ed. Londres/Nova York: Routledge, 2004. p. 180-192.

LEIMONTAS, Renan Abdalla. Eu vou equalizar sua cara: análise da localização do video game Mortal Kombat X. 2017. 136f. Mestrado (Estudos da Tradução) - Centro de Comunicação e Expressão, Universidade Federal de Santa Catarina, Florianópolis, 2017.

LONG, Jixing. Translation definitions in different paradigms. Canadian Social Science, Québec, v. 9, n. 4, p. 107-115, 2013.

MALTA, Gleiton; BARCELLOS, Luiz Gustavo Nogueira. A localização em jogo na tradução de games. Tradterm, v. 32, p. 116-137, 2018. doi: https://doi.org/10.11606/issn.23179511.v32i0p116-137

NEWMARK, Peter. A textbook of translation. New York: Prentice Hall, 1988.

NOVAK, Jeannie. Game development essentials. 3. ed. Clifton Park: Delmar Cengage Learning, 2012.

BARCELOS, Luiz Gustavo Nogueira; MALTA, Gleiton. A tradução/localização de videogames: Um mapeamento das pesquisas realizadas em instituições de ensino superior brasileiras entre 1998 e 2018 . Belas Infiéis, Brasília, v. 9, n. 4, p. 127 144, jul./set., 2020. e-ISSN: 2316-6614. DOI: doi.org/10.26512/belasinfieis.v9.n4.2020.27155 
PACHECO, Paula. Mercado nacional de games deve movimentar mais de US\$ 1,5 bilhão em 2018. Correio Braziliense, 24 jul. 2018. Disponível em: https://www.correiobraziliense.com.br/app/noticia/economia/2018/07/24/internas_economia,6 96929/mercado-nacional-de-games-deve-movimentar-mais-de-us-1-5-bilhao-em-20.shtml.

Acesso em: 2 mar. 2020.

PAGANO, Adriana S.; VASCONCELLOS, Maria Lúcia. Estudos da tradução no Brasil: reflexões sobre teses e dissertações elaboradas por pesquisadores brasileiros nas décadas de 1980 e 1990. D.E.L.T.A., São Paulo, v. 19, n. spe, p. 1-25, 2003. doi: https://doi.org/10.1590/S0102-44502003000300003

PETRÓ, Gustavo. No Brasil, empresas investem em jogos traduzidos para o português. Gl, 19 mar. 2011. Disponível em: http:/g1.globo.com/tecnologia/noticia/2011/03/no-brasilempresas-investem-em-jogos-traduzidos-para-o-portugues.html. Acesso em: 2 mar. 2020.

ROLLINGS, Andrew; MORRIS, David. Game architecture and design: a new edition. Indianapolis: New Riders, 2004.

SCHÄLER, Reinhard. Localization. In: BAKER, Mona (Ed.). Routledge encyclopedia of translation studies. London/New York: Routledge, 1998. p. 157-161.

SILVA, Hemanoel Mariano Sousa e. O estado da arte da localização/tradução de videogames. 2018. 82f. Dissertação (Mestrado em Estudos da Tradução) - Universidade Federal do Ceará, Fortaleza (CE), 2018.

SILVA, Fernando. The mapping of localized contents in the videogame inFamous 2: a multimodal corpus-based analysis. 2016. 307f. Doutorado (Estudos da Tradução) - Centro de Comunicação e Expressão, Universidade Federal de Santa Catarina, Florianópolis, 2016.

SOUZA, Ricardo Vinicius Ferraz. Tradução e videogames: uma perspectiva históricodescritiva sobre a localização de games no Brasil. 2015. 395f. Dissertação (Mestrado em Estudos da Tradução) - Faculdade de Filosofia, Letras e Ciências Humanas, Universidade de São Paulo, 2015.

VIDEOGAMES fazem frente à poderosa indústria cinematográfica. Correio Braziliense, 30 jul. 2015. Disponível em: https://www.correiobraziliense.com.br/app/noticia/diversao-earte/2015/07/30/interna_diversao_arte,492553/videogames-fazem-frente-a-poderosaindustria-cinematografica.shtml Acesso em: 2 mar. 2020.

\footnotetext{
${ }^{1}$ Tradução nossa: "Se eu vendo para você, eu falo sua língua. Se eu estou comprando, então é você que tem de falar alemão!"

2 Texto original: "[...] it is rendering the meaning of a text into another language in the way that the author intended the text".

${ }^{3}$ Tradução nossa para: "[...] can be defined as the linguistic and cultural adaptation of digital content to the requirements and locale of a foreign market, and the provision of services and technologies for the management of multilingualism across the digital global information flow."

4 A fim de diferenciação conceitual, as expressões "tradução" e "localização" - assim como suas respectivas variações, serão tratadas de maneira distinta; embora haja uma nítida conexão entre ambas.
} 


\footnotetext{
${ }^{5}$ Disponível em: dgp.cnpq.br/dgp/espelhogrupo/2060501981311209. Último acesso em: 11 set. 2019.

${ }^{6}$ Com base em dados de pesquisa realizada pela Newzoo (empresa especializada em pesquisa no mercado de games), "a indústria dos jogos eletrônicos deve arrecadar, até o fim deste ano [2015], US\$ 91 bilhões, enquanto o consumidor irá gastar US\$ 39,1 bilhões com cinema, de acordo com dados da pesquisa da PwC" (VIDEOGAMES..., 2015).

7 Tradução nossa para: "Some games during this decade pushed the boundaries proving beyond any doubts what could be done in localisation. Baldur's Gate (Bioware/Interplay 1998), for example, was oneof the first role playing games to be 'fully' translatedand dubbed into Spanish. It was especiallymeaningful from the localisation point of viewbecause, like most RPGs, it had hundreds ofthousands of words (part of books, maps, andparchments found in the adventure) to betranslated, as well as several thousand voiceoverfiles to be recorded for each language version.By the end of the nineties revenues had doubledin the games industry (ESA), and althoughthere was some internal growth more than halfof it came from the efforts put into localisation..This tendency continued in the following decade".

${ }^{8}$ Os links para o acesso dos repositórios de cada uma das instituições em questão são fornecidos no capítulo intitulado "Apresentação de Dados".

${ }^{9}$ Optamos por utilizar as variadas possibilidades em que a expressão "videogame" poderia ocorrer porque era esperado que os(as) autores(as) tivessem diferentes preferências por variações específicas.

${ }^{10}$ Cf. Bernal-Merino (2011).

${ }^{11}$ Magic: The Gathering é um jogo de cartas (ou cards) colecionáveis e é o precursor do seu gênero. O jogo foi criado por Richard Garfield e teve sua primeira publicação em 1993.

${ }^{12}$ Cf. Petró (2011).

${ }^{13}$ Cf. Pacheco (2018).

${ }^{14}$ Cf. Leimontas (2017).
}

\section{NOTA DOS AUTORES}

144 Luiz Gustavo Nogueira BARCELOS - Mestrando em Estudos da Tradução pela Universidade de Brasília. Graduado em Letras Tradução-Espanhol (2017) pela mesma instituição. Universidade de Brasília, Instituto de Letras, Programa de Pós-Graduação em Estudos da Tradução. Brasília, Distrito Federal, Brasil.

ORCID: https://orcid.org/0000-0002-2868-9626

Currículo acadêmico: http://lattes.cnpq.br/5802107363580035

E-mail: barcelos_gustavo@hotmail.com

Gleiton MALTA - Professor adjunto na Universidade de Brasília. Doutor em Estudos Linguísticos (2015) pela Universidade Federal de Minas Gerais. Mestre em Linguística Aplicada (2009) pela Universidade de Brasília. Graduado em Letras-Espanhol (2001) pela mesma instituição. Universidade de Brasília, Instituto de Letras, Departamento de Letras Estrangeiras e Tradução. Brasília, Distrito Federal, Brasil.

ORCID: https://orcid.org/0000-0003-0359-4046

Currículo acadêmico: http://lattes.cnpq.br/0525598075310277

E-mail: gleitonmalta@gmail.com 